\title{
Euler Diagrams Through the Looking Glass: From Extent to Intent
}

\author{
Gem Stapleton ${ }^{1}$, Amirouche Moktefi $^{2}$, John Howse $^{1}$, and Jim Burton ${ }^{1}$ \\ 1 Centre for Secure, Intelligent and Usable Systems, University of Brighton, UK \\ \{g.e.stapleton, john.howse, j.burton\}@brighton.ac.uk \\ 2 Tallinn University of Technology, Estonia \\ amirouche.moktefi@ttu.ee
}

\begin{abstract}
Extension and intension are two ways of indicating the fundamental meaning of a concept. The extent of a concept, $C$, is the set of objects which correspond to $C$ whereas the intent of $C$ is the collection of attributes that characterise it. Thus, intension defines the set of objects corresponding to $C$ without naming them individually. Mathematicians switch comfortably between these perspectives but the majority of logical diagrams deal exclusively in extension. Euler diagram indicate sets using curves to depict their extent in a way that intuitively matches the relations between the sets. What happens when we use spatial diagrams to depict intension? What can we infer about the intension of a concept given its extension, and vice versa? We present the first steps towards addressing these questions by defining extensional and intensional Euler diagrams and translations between the two perspectives. We show that translation in either direction leads to a loss of information, yet preserves important semantic properties. To conclude, we explain how we expect further exploration of the relationship between the two perspectives could shed light on connections between diagrams, extension, intension, and well-matchedness.
\end{abstract}

\section{Introduction}

A general term (e.g. "country", "circle", "horse", etc.) is commonly understood to refer to a collection of individuals who share one or more attributes. The set of individuals to which the term refers is called its extension (or extent), while the set of attributes shared by those individuals is called the intension (or intent) of the term. The trio (term, intent, extent) and its structure can be represented by a triangle (Figure 1). Various names have been used in logic literature to capture this distinction. Intent is sometimes referred to as the connotation of a term while extent is said to be its denotation. Hence, a term is said to connote its intent and denote its extent. These two names can be used to characterize the two sides of our triangle that connect a term to its intent and extent. The object of this paper is to diagrammatically investigate the third side of the triangle which depicts the relation between the intent and the extent of a given term.

To illustrate, the diagram in figure 2 represents three sets containing invididuals: People $(P)$, Children $(C)$, and Dogs $(D)$. It therefore presents an 


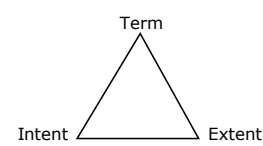

Fig. 1. Term-Intent-Extent.

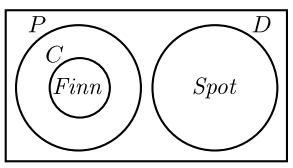

Fig. 2. Extension.

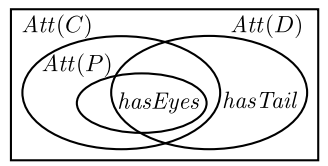

Fig. 3. Intension.

extensional view. The set of People, for example, comprises the individuals that have the attributes that define what it means to be a person. By contrast, figure 3 presents an intensional view, representing the sets of attributes of people, $\operatorname{Att}(P)$ and so forth. We can see, from figure 3, that all children have all of the person attributes, including having eyes. Moreover, dogs possess attributes that no child or person has, such as having a tail. So, the dog Spot has a tail but the child Finn does not.

The distinction between intent and extent (known under various denominations) has played a significant but often undervalued role in the development of modern logic. Although it is sometimes traced in earlier writings, the distinction itself is often attributed to Antoine Arnauld and Pierre Nicole's Logique de Port Royal (1662). Since then, logicians who have designed logical calculi oscillated between the two interpretations. For instance, Gottfried Leibniz generally favoured the intensional interpretation while George Boole privileged the extensional interpretation, with various motivations being offered to justify the superiority of each view over the other [3]. In his 1918 survey of symbolic logic, C. I. Lewis explained the successes of Boole and his (mainly English-speaking) followers by their appeal to extent unlike their (German-speaking) predecessors who favored intent [6, pp 35-37]. Although Gottlob Frege's logic was primarily intensional, John Venn declared in 1894 that "the true intensive view is practically abandoned now, though verbally it is from time to time espoused" [13, p 453]. Extensional logics apparently became dominant at the beginning of the twentieth century. Intensional logic was then generally believed to be at best cumbersome, if not entirely impossible [2, p 387] [9, p 141]. The rise and fall of intensional logics can also be traced in the development of logic diagrams. Indeed, Leibniz and several of his followers aimed at a scheme that could stand within both views, depending on whether it was the intent or the extent that was represented [1]. However, extensional diagrams shortly became dominant as geometrical relations of the diagrams appeared to match better with the logical relations of the extents than those of the intents [7]. Despite the declared superiority of extensional logic, interest in intensional logics has resurfaced in the twentieth century, notably in the footsteps of Alonzo Church and Rudolph Carnap [4]. Today, the distinction between intent and extent is commonplace and is frequently met with in modern logic textbooks [5, pp 91-94].

There are several difficulties in determining precisely what the intent and the extent of a term involve and logicians have long considered various views on this question. Regarding intent, an objective standpoint includes any attribute possessed by the individuals to whom the term refers, whether that attribute is 
known or unknown to the people who use the term. A subjective view, on the other hand, supports the claim that intent should include merely the attributes that come to the mind of those who use the term. However, since different people might have different collections of attributes in mind, logic textbooks often adopt a conventional attitude in which the intent of a term refers to what is commonly attributed to it. The description of the extent of a term also involves some complications. A major difficulty concerns the definition of what counts as an individual. If we are to determine the extent of the concept "animal", should one list general species (elephants, penguins, sharks, etc.) or rather point to every specific animal considered individually? The latter technique often leads to long or infinite enumerations while the former has obvious practical advantages, although it does requires the formation of sub-classes.

The relation between intent and extent is also complex. It is first noted that, though the intent of a term might remain fixed, extent can change over time. The extent of the term "President of France" regularly changes when elections introduce new individuals with the salient attributes. Extents might also be empty; we may think, for instance, of the term "current king of France". However, equivalent extents do not necessarily indicate equivalence of intents. For example, there are various sets of attributes that can be offered to form the intent of the term whose extent contains the individuals Spain and Portugal. One might think of the term as those countries through which the Douro River flows. Alternatively, the term could be thought of as the countries through which the Tagus River flows. It could also be said that they are the last two winners to-date of the UEFA European Championship. All these intensional definitions denote the same extent, $\{$ Spain, Portugal $\}$.

The relation between extent and intent is usually addressed through the principle of their inverse variation, whereby increasing the intent of a term by adding an attribute to it generally entails a decrease of its extent. If one thinks of the intent of the term "triangle" and adds to it the attribute of being isosceles, we remove from the extent of this term all the triangles which do not have the latter attribute (i.e. are not isosceles). Hence, the increase of intent produced a decrease of extent. In this example, the intent of a triangle is included in that of an isosceles triangle. Yet, it is the inverse that is observed for the extents, since the extent of isosceles triangles is included within the extent of triangles. It might be that an increase of intent does not produce a decrease of extent. This is the case for instance if one adds the attribute of being crossed by the Douro River to the intent whose attributes define the countries which are crossed by the Tagus River. The extent, $\{$ Spain, Portugal $\}$, remains the same. Similarly, if a given extent is empty it does not decrease if new attributes are added to its intent. Still, in all these examples, when intent increases, extent is observed to decrease or remain stable, but definitely not increase. Similar principles are found for decreasing intents and for comparable changes in the scope of an extent.

As interesting as these principle can be, they only account for the specific case where a term is increased (respectively, decreased), meaning that it is entirely included (respectively, includes) another term. In the following, we attempt a 
more systematic consideration of the relations between intent and extent. In the next section we present two systems of Euler diagrams with $\otimes$-sequences: one in which curves denote the extent of terms (as is usually the case), and one in which curves denote intent. In section 3 we establish a connection between interpretations of extensional and intensional diagrams, linking the semantics of the two systems through a relation that embodies the notion of one interpretation being respectful of another. In sections 4 and 5 we define translations from extensional and intensional diagrams to their respective counterparts. We show that these translations necessarily involve the loss of information yet preserve important semantic properties. Finally, we conclude with some thoughts on the implications for this work.

\section{Syntax and Semantics}

We follow a standard approach to formalizing the syntax and semantics of Euler diagrams containing $\otimes$-sequences (see Stapleton [11] for an overview of formalization techniques). We illustrate the key ideas via the example in figure 4 . This diagram contains three curves, each of which has a label. The curves give rise to zones: a zone is a region of the plane inside some (possibly no) curves and outside the remaining curves. There are four zones inside this diagram, such as the one inside just the curve $P$ but outside $Q$ and $R$, and another zone outside all three curves. Sometimes, zones are shaded. In this example, the zone inside $R$ but outside $P$ and $Q$ is shaded. There are two $\otimes$-sequences. One of them is inside a single zone and the other comprises two $\otimes$ symbols joined by a line.

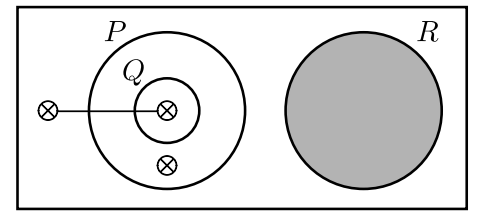

Fig. 4. An Euler diagram with $\otimes-$ sequences.

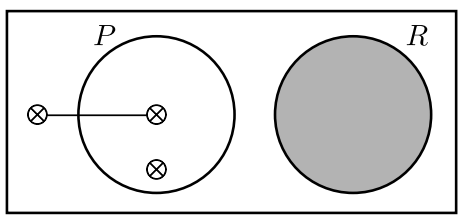

Fig. 5. Removing a curve: zonal regions.

We formalize diagrams at the abstract syntax level. Curves can be identified by their labels which are chosen from some given set $\mathcal{L}$. Zones are then formally defined as a pair of disjoint sets of labels, $\left(L_{i}, L_{o}\right)$. In figure 4 , the four zones are $(\emptyset,\{P, Q, R\}),(\{P\},\{Q, R\}),(\{P, Q\},\{R\})$ and, lastly, the shaded zone $(\{R\},\{P, Q\})$. The formalization of the $\otimes$-sequences is similar: they are identified at the abstract level by the set of zones in which they are placed. In our example, the two $\otimes$-sequences are $\{(\{P\},\{Q, R\})\}$ and $\{(\emptyset,\{P, Q, R\}),(\{P, Q\},\{R\})\}$. We now provide a formal definition of these important concepts.

Definition 1. Given $\mathcal{L}$, we define a zone over $\mathcal{L}$ to be a pair, $\left(L_{i}, L_{o}\right)$, where $L_{i} \cup L_{o} \subseteq \mathcal{L}$, and $L_{i} \cap L_{o}=\emptyset$. The set of all zones formed from $\mathcal{L}$ is denoted 
$\mathcal{Z}_{\mathcal{L}}$. A set of zones formed over $\mathcal{L}$ is called a region formed over $\mathcal{L}$ and the set of all regions is denoted $\mathcal{R}_{\mathcal{L}}$.

It is notable that the above definition makes the set of labels, $\mathcal{L}$, over which zones and regions are formed explicit. Later, we will be working with two distinct systems of diagrams that draw their labels from distinct sets. So it is important to be clear about over which label set a diagram and its components are formed.

Definition 2. An Euler diagram with $\otimes$-sequences, $d_{\mathcal{L}}$, formed over $\mathcal{L}$ is a tuple $d_{\mathcal{L}}=\left(L, Z, Z^{*}, S\right)$ where

1. $L$ is a finite subset of $\mathcal{L}$.

2. $Z$ is a set of zones such that for each zone, $\left(L_{i}, L_{o}\right)$, in $Z, L_{i} \cup L_{o}=L$,

3. $Z^{*}$ is a subset of $Z$ whose elements are called shaded zones, and

4. $S$ is a set of regions that identify the $\otimes$-sequences: $S \subseteq \mathbb{P} Z \backslash\{\emptyset\}$.

We will sometimes simply say Euler diagram with $\otimes$-sequences or $\mathcal{L}$-diagram, in place of Euler diagram with $\otimes$-sequences formed over $\mathcal{L}$. Furthermore, we will similarly omit saying 'formed over $\mathcal{L}$ ' when referring to other syntactic items.

Our attention now turns to semantics. Referring again to figure 4, intuitively this diagram tells us that $Q \subseteq P$ and $P \cap R=\emptyset$ due to the spatial relationships between the curves. The shading is used in the same fashion as for Venn diagrams [12], and as seen in Shin's Venn-I and Venn-II systems [10]: shaded zones represent empty sets. So, the set $R$ is empty in our example. Again, also following Shin's use of $\otimes$-sequences, regions containing an entire $\otimes$-sequence are non-empty (see Moktefi and Pietarinen [8] for the origins and development of this notation). So, we see that $P \backslash(Q \cup R) \neq \emptyset$ and, taking the universal set to be $\mathcal{U},(\mathcal{U} \backslash(P \cup Q \cup R)) \cup((P \cap Q) \backslash R) \neq \emptyset$. Given these insights, we proceed with our formalization following a standard model-theoretic approach. In our case, labels are interpreted as sets, which we then extend to interpret zones and regions:

Definition 3. An interpretation over $\mathcal{L}$, denoted $\mathcal{I}_{\mathcal{L}}$, is a pair, $\mathcal{I}_{\mathcal{L}}=(\mathcal{U}, \Psi)$, where $\mathcal{U}$ is a set and $\Psi$ is a function, $\Psi: \mathcal{L} \cup \mathcal{Z}_{\mathcal{L}} \cup \mathcal{R}_{\mathcal{L}} \rightarrow \mathbb{P U}$, mapping labels, zones and regions to sets such that

1. for each zone, $\left(L_{i}, L_{o}\right)$,

$$
\Psi\left(L_{i}, L_{o}\right)=\left(\bigcap_{l \in L_{i}} \Psi(l)\right) \backslash\left(\bigcup_{l \in L_{o}} \Psi(l)\right) \text {, and }
$$

2. for each region, $r, \Psi(r)=\bigcup_{z \in r} \Psi(z)$.

The last remaining consideration, when defining the semantics, is to provide conditions under which an interpretation 'agrees' with the intended meaning of the diagram. We have already seen that shaded zones represent empty sets and that regions containing entire $\otimes$-sequences represent non-empty sets. Additionally, the relationship between the curves (in a drawn diagram) is entirely captured by the set of zones (at the abstract level). In particular, between them, all of the (abstract) zones must represent the universal set. If these three conditions are all met then the interpretation is a model: 
Definition 4. Given an Euler diagram with $\otimes$-sequences, $d_{\mathcal{L}}=\left(L, Z, Z^{*}, S\right)$, and an interpretation, $\mathcal{I}_{\mathcal{L}}=(\mathcal{U}, \Psi)$, we say that $\mathcal{I}$ is a model for d provided

1. between them, the zones in $d_{\mathcal{L}}$ represent the universal set: $\Psi(Z)=\mathcal{U}$,

2. the shaded zones in $d_{\mathcal{L}}$ represent the empty set: $\Psi\left(Z^{*}\right)=\emptyset$, and

3. each $\otimes$-sequence is placed in a region that represents a non-empty set: for all $r$ in $S, \Psi(r) \neq \emptyset$.

Having now defined the syntax and semantics, we introduce several further syntactic notions that will be of use later. The first focuses on the zones that are not present, (called missing zones), given the labels used in a diagram:

Definition 5. Let $d_{\mathcal{L}}=\left(L, Z, Z^{*}, S\right)$ be an Euler diagram with $\otimes$-sequences. The missing zones of $d_{\mathcal{L}}$ are elements of

$$
\mathcal{M Z}\left(d_{\mathcal{L}}\right)=\left\{\left(L_{i}, L_{o}\right) \in \mathcal{Z}_{\mathcal{L}}: L=L_{i} \cup L_{o}\right\} \backslash Z .
$$

In our running example, figure 4, there are four missing zones:

$$
\mathcal{M Z}\left(d_{\mathcal{L}}\right)=\{(\{Q\},\{P, R\}),(\{P, R\},\{Q\}),(\{Q, R\},\{P\}),(\{P, Q, R\}, \emptyset)\} .
$$

Just as zones play a central role in our understanding of Euler diagrams, so too do regions that become zones when curves are removed. Figure 5 shows the result of removing $Q$ from figure 4 . The zone inside just $P$ in figure 5 arises from two zones in figure 4 . Regions that become zones when curves are removed are called zonal regions and, just like zones, can be identified by the curves that contain the region and those which do not contain the region:

Definition 6. Let IN, OUT and L be sets of labels drawn from $\mathcal{L}$. A zonal region given IN, OUT and $L$ is a set of zones, denoted $\langle I N, O U T, L\rangle$, where

$$
\langle I N, O U T, L\rangle=\left\{\left(L_{i}, L_{o}\right): I N \subseteq L_{i} \wedge O U T \subseteq L_{o} \wedge L=L_{i} \cup L_{o}\right\} .
$$

For example, in figure 4, the zonal region inside $P$ but outside $R$ is given by

$$
\langle\{P\},\{R\},\{P, Q, R\}\rangle=\{(\{P\},\{Q, R\}),(\{P, Q\},\{R\})\} .
$$

Having formally defined the syntax and semantics of Euler diagrams with $\otimes$ sequences, and various related notions, we are in a position to explore extensional and intensional viewpoints using Euler diagrams.

\section{Sets of Individuals and Their Attributes}

Euler diagrams are typically used to represent sets containing individuals and, therefore, visualize an extensional view of the world. An intensional viewpoint, however, considers the attributes that characterise the individuals in sets. Consider the example in figure 6 . The diagram $d_{\mathcal{E} \mathcal{X}}$ represents sets of individuals who are members of four sports clubs and, so, is extensional. We can see that 
everyone who is in the Triathlon club (represented by the curve labelled $T$ ) is also a member of the Swimming club. However, nobody in the Swimming club is in either the Cycling or Football clubs. Each club imposes the condition on its members that they must be active participants in the relevant sport; so, members of the swimming club must be able to swim, and those in the cycling and football clubs are all cyclists and, respectively, footballers. Individuals who are members of the Triathlon club have the attributes of being a cyclist, a swimmer and a runner, as well as the attribute of Triathlon club membership. As it happens, all members of the Cycling club are active runners. These sets of attributes therefore correspond to the intensional viewpoint. Therefore, $d_{\mathcal{I N}}$ (where $\operatorname{Int}(T)$ is the set of attributes that characterise set $T$ and so forth), represents the relationships between the attributes possessed by the individuals in the sets represented by $d_{\mathcal{E} \mathcal{X}}$, given the particular situation just described.
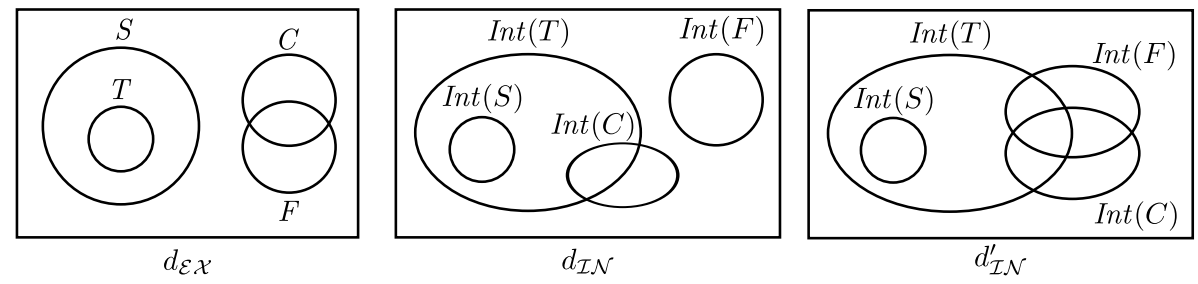

Fig. 6. Sports clubs and their members' attributes.

Fig. 7. Alternative attributes.

Whilst this example is suitable for providing intuition about the relationship between individuals and their attributes, the diagram $d_{\mathcal{I N}}$ just derived was, we emphasise, particular to the interpretation given. We could have had an alternative situation where all of the footballers are runners and cyclists but are not members of the triathalon or swimming clubs since none of them is able to swim. This situation would give rise to the diagram in figure 7. Our goal is to define a respectful translation from diagrams representing individuals to diagrams representing their attributes that ensures their models correspond entirely. Likewise, we also seek a respectful translation from diagrams representing attributes to diagrams representing individuals so that their models correspond. The purpose of this section is to set up a framework that allows these translations to be defined formally and for us to establish that the resulting diagrams' model sets correspond in an appropriate way.

To this end, we investigate two parallel systems of Euler diagrams with $\otimes$ sequences. We define the first system to be formed over a set of labels that we call $\mathcal{E} \mathcal{X}$ (so $\mathcal{E} \mathcal{X}$ is a particular choice of $\mathcal{L}$ ), whose elements are called extensional labels. Further, we define the second system to be formed over a set of labels that we call $\mathcal{I N}$, whose elements are called intensional labels. Importantly, we assume that $\mathcal{E} \mathcal{X}$ and $\mathcal{I N}$ are disjoint and have the same cardinality. We now syntactically link these two systems. 
Definition 7. A bijective function Int: $\mathcal{E} \mathcal{X} \rightarrow \mathcal{I N}$, which maps each extensional label to an intensional label is called an intensional label allocation function. The function Ext: $\mathcal{I N} \rightarrow \mathcal{E X}$ is the inverse of Int.

From this point forward, we assume an intensional label allocation function, Int, has been defined but we also need a semantic link. $\mathcal{E} \mathcal{X}$-diagrams and $\mathcal{I N}$ diagrams are taken to have semantics where the universal sets contain individuals and, respectively, attributes. To this end, the set of individuals is denoted $\mathcal{I N D}$ and the attributes $\mathcal{A T} \mathcal{T}$. Consequently, for example, given $\mathcal{I}_{\mathcal{E X}}=(\mathcal{U}, \Psi)$, we have $\mathcal{U} \subseteq \mathcal{I N D}$. All interpretations over $\mathcal{E} \mathcal{X}$ have universal sets that are subsets of $\mathcal{I N D}$ whereas those over $\mathcal{I N}$ have universal sets that are subsets of $\mathcal{A T} \mathcal{T}$. As our intention is to explore the relationship between information about individuals and information about their attributes, we further define a function between the sets $\mathcal{I N D}$ and $\mathcal{A T} \mathcal{T}$ to formalize this notion.

Definition 8. A function, att: $\mathcal{I N D} \rightarrow \mathbb{P}(\mathcal{A T} \mathcal{T})$ is called an attribute identification function.

As with the function Int, we assume from this point forward that a specific att is given. We now use att to define a link between interpretations, thus linking the semantics of the two systems:

Definition 9. Let $\mathcal{I}_{\mathcal{E} \mathcal{X}}=\left(\mathcal{U}_{\mathcal{E} \mathcal{X}}, \Psi_{\mathcal{E} \mathcal{X}}\right)$ and $\mathcal{I}_{\mathcal{I N}}=\left(\mathcal{U}_{\mathcal{I N}}, \Psi_{\mathcal{I N}}\right)$ be interpretations over $\mathcal{E} \mathcal{X}$ and $\mathcal{I N}$ respectively. We say that $\mathcal{I}_{\mathcal{I N}}$ is respectful of $\mathcal{I}_{\mathcal{E} \mathcal{X}}$ and att provided for all $i \in \mathcal{U}_{\mathcal{E} X}$, and for all $P \in \mathcal{E} \mathcal{X}$

$$
i \in \Psi_{\mathcal{E X}}(P) \Leftrightarrow \operatorname{att}(i) \supseteq \Psi_{\mathcal{I N}}(\operatorname{Int}(P)) .
$$

This definition is illustrated in figure 8 , in the case where $\Psi_{\mathcal{E} \mathcal{X}}(P)$ is not empty (it contains $i_{1}$ ) and neither is is complement (which contains $i_{2}$ ). The attributes of individual $i_{1}$ are $\operatorname{att}\left(i_{1}\right)$ and, intuitively, since $i_{1}$ is in $P$ (blurring the distinction between syntax and semantics), $i_{1}$ must have all of the attributes that are required of individuals in $P$. In figure 8 , this is visually indicated by the arrow from $i_{1}$ targeting a superset of $\Psi_{\mathcal{I N}}(\operatorname{Int}(P))$. Likewise, the individual $i_{2}$ must be missing an attribute, say $a$, that characterises $P$. From this point forward, in general we will refer to the attributes that characterise $P$ as $P$ attributes and so forth.

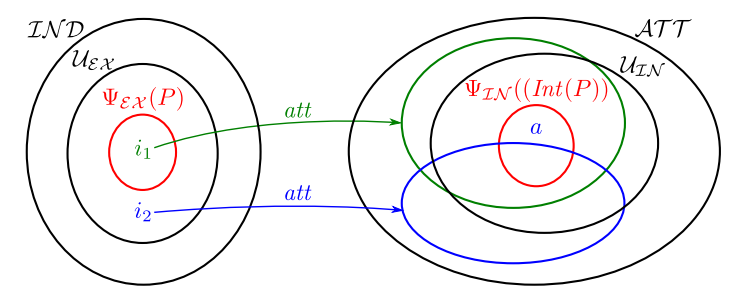

Fig. 8. Illustrating respectful interpretations. 


\section{From Extensional to Intensional Diagrams}

Consider the example in figure 9, where $d_{\mathcal{E} \mathcal{X}}$ indicates that $P \backslash(Q \cup R)$ is nonempty using an $\otimes$-sequence. Therefore, we know that there is an element in $P$ that has all of the $P$-attributes but is missing at least one of the $Q$-attributes and at least one of the $R$-attributes. So, more formally, given a model $\mathcal{I}_{\mathcal{E} \mathcal{X}}=$ $\left(\mathcal{U}_{\mathcal{E} \mathcal{X}}, \Psi_{\mathcal{E} \mathcal{X}}\right)$ for $d_{\mathcal{E} \mathcal{X}}, \Psi_{\mathcal{E} \mathcal{X}}(P)$ contains an individual, say $i$, that is not in $\Psi_{\mathcal{E} \mathcal{X}}(Q)$ nor in $\Psi_{\mathcal{E X}}(R)$.

Moreover, given a respectful interpretation, $\mathcal{I}_{\mathcal{I N}}=\left(\mathcal{U}_{\mathcal{I N}}, \Psi_{\mathcal{I N}}\right)$, att $(i)$ ensures $\operatorname{att}(i) \supseteq \Psi_{\mathcal{I N}}(\operatorname{Int}(P))$, att $(i) \nsupseteq \Psi_{\mathcal{I N}}(\operatorname{Int}(Q))$ and $\operatorname{att}(i) \nsupseteq \Psi_{\mathcal{I N}}(\operatorname{Int}(R))$. From att $(i) \nsupseteq \Psi_{\mathcal{I N}}(\operatorname{Int}(Q))$ we can deduce that there is a $Q$-attribute that is not a $P$-attribute. Likewise, there is an $R$-attribute that is not a $P$-attribute, which may or may not be the same as the $Q$-attribute. This situation is captured by $d_{\mathcal{I N}}$, where two $\otimes$-sequences are placed in zonal regions. For instance, one of the $\otimes$-sequences is in $\langle\{\operatorname{Int}(Q)\},\{\operatorname{Int}(P)\},\{\operatorname{Int}(P), \operatorname{Int}(Q), \operatorname{Int}(R)\}\rangle$ (i.e. the $\otimes$-sequence is inside $\operatorname{Int}(Q)$ but outside $\operatorname{Int}(P))$.
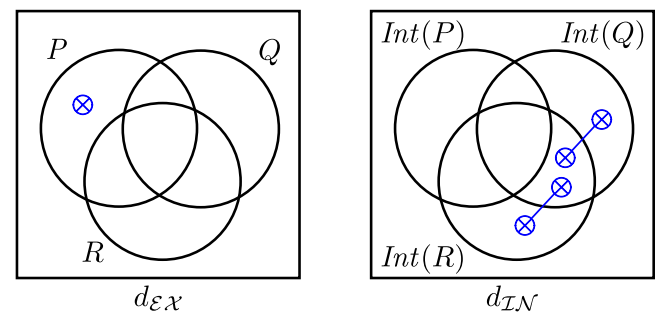

Fig. 9. A respectful translation from extensional to intensional diagrams.

In general, given a single $\otimes$ placed in a zone, $\left(L_{i}, L_{o}\right)$, of an $\mathcal{E} \mathcal{X}$-diagram we know that there is an element (in the set represented by) $\left(L_{i}, L_{o}\right)$; in what follows we frequently blur the distinction between syntax and semantics as we have just done here. This element has all of the attributes in (the sets denoted by the) intensional labels arising from $L_{i}$; in our previous example, the $\otimes$-sequence in $d_{\mathcal{E} \mathcal{X}}$ was in the zone $(\{P\},\{Q, R\})$ and, informally, had all the $P$-attributes. Importantly, such an element is missing at least one attribute from each of the intensional labels arising from $L_{o}$; in the previous example, informally, the $\otimes$ sequence in $d_{\mathcal{E} \mathcal{X}}$ was missing a $Q$-attribute and an $R$-attribute. This means we know that each intensional label arising from $L_{o}$ contains an attribute that is not in any of the intensional labels arising from $L_{i}$. This leads to our next definition which identifies, for any zone, a corresponding zonal region such that if the zone in a $P$-diagram contains an $\otimes$ then the zonal region will contain an $\otimes$-sequence:

Definition 10. Let $\left(L_{i}, L_{o}\right)$ be a zone formed over $\mathcal{E} \mathcal{X}$. Let $\langle I N, O U T, I\rangle$ be a zonal region formed over $\mathcal{I N}$. Then $\langle I N, O U T, I\rangle$ is a corresponding $\mathcal{I N}$ region of $\left(L_{i}, L_{o}\right)$ provided 
1. IN contains a single intensional label arising from $L_{o}$ :

$$
I N=\{\operatorname{Int}(p)\} \text { for some } p \in L_{o}
$$

2. OUT contains the intensional labels arising from $L_{i}$ :

$$
O U T=\left\{\operatorname{Int}(p): p \in L_{i}\right\}, \text { and }
$$

3. I contains the intensional labels arising from $L_{i} \cup L_{o}$ :

$$
I=\left\{\operatorname{Int}(p): p \in L_{i} \cup L_{o}\right\}
$$

Given $\left(L_{i}, L_{o}\right)$, the set of zonal regions which are corresponding $\mathcal{I N}$-regions is denoted $Z R\left(L_{i}, L_{o}\right)$.

Referring again to figure 9 , given $I=\{\operatorname{Int}(P), \operatorname{Int}(Q), \operatorname{Int}(R)\}$, we have

$$
Z R(\{P\},\{Q, R\})=\{\langle\{\operatorname{Int}(Q)\},\{\operatorname{Int}(P)\}, I\rangle,\langle\{\operatorname{Int}(R)\},\{\operatorname{Int}(P)\}, I\rangle\} .
$$
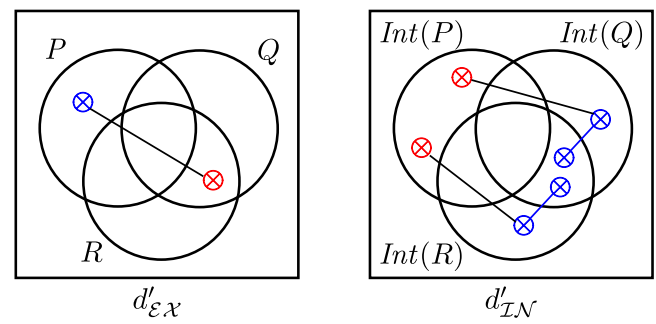

Fig. 10. A more complex $\otimes$-sequence case.

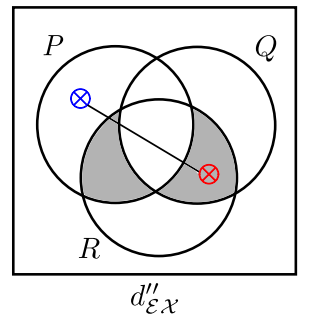

Fig. 11. The impact of shading.

Whilst this gives us insight into how to translate the information provided by a single $\otimes$-sequence placed in a zone, we need to consider the more general case where $\otimes$-sequences are placed in multiple zones. Extending the example in figure 9 to figure 10 , we obtain $d_{\mathcal{I} \mathcal{N}}^{\prime}$ from $d_{\mathcal{E} \mathcal{X}}^{\prime}$. Here, we have $Z R(\{P\},\{Q, R\})$ (as given above) from the (blue) $\otimes$ in $(\{P\},\{Q, R\})$ and

$$
Z R(\{Q, R\},\{P\})=\{\langle\{\operatorname{Int}(P)\},\{\operatorname{Int}(Q), \operatorname{Int}(R)\},\{\operatorname{Int}(P), \operatorname{Int}(Q), \operatorname{Int}(R)\}\rangle\}
$$

from the $($ red $) \otimes$ in $(\{Q, R\},\{P\})$. The blue $\otimes \mathrm{s}$ in $d_{\mathcal{I N}}^{\prime}$ arise from the blue $\otimes$ in $d_{\mathcal{E} \mathcal{X}}^{\prime}$. Likewise for the red $\otimes$ s. Given that $\otimes$-sequences provide disjunctive information, there are a range of possibilities for the presence of attributes as shown in $d_{\mathcal{I N}}^{\prime}$; essentially, the $\otimes$-sequences in $d_{\mathcal{I N}}^{\prime}$ capture this range of possibilities in a conjunctive normal form. Definition 11 makes this insight precise, where $r=\left\{z_{1}, \ldots, z_{n}\right\}$ can be thought of as a region containing an entire $\otimes$-sequence: 
Definition 11. Let $r=\left\{z_{1}, \ldots, z_{n}\right\}$ be a region formed over $\mathcal{E} \mathcal{X}$. The elements of the set of regions, $R(r)$, given by

$$
R(r)=\left\{z r_{1} \cup \ldots \cup z r_{n}: z r_{1} \in Z R\left(z_{1}\right) \wedge \ldots \wedge \cup z r_{n} \in Z R\left(z_{n}\right)\right\}
$$

are corresponding $\mathcal{I N}$-regions of $r$.

Having considered the presence of $\otimes$-sequences in $\mathcal{E} \mathcal{X}$-diagrams, our attention now turns to shading. In figure 11, shading has been placed in the zones $(\{Q, R\},\{P\})$ and $(\{P, R\},\{Q\})$. This provides information beyond the $\otimes$-sequence, such as that $(\{Q, R\},\{P\})$ represents the empty set. Therefore, from the $\otimes$-sequence and the shading, we know that $(\{P\},\{Q, R\})$ represents a non-empty set. Thus, the information we gain about the presence of attributes arising from the $\otimes$-sequence in $d_{\mathcal{E} \mathcal{X}}^{\prime \prime}$ reverts to what we found in figure 9 . Consider now the shading in $(\{P, R\},\{Q\})$. This shading tells us that there are no elements in both $P$ and $R$ but outside $Q$ but does not provide any information about attributes.

Importantly, it is true in general that the absence of individuals in a set does not provide any information about the absence of attributes. This is a major point: shading in $\mathcal{E} \mathcal{X}$-diagrams does not provide information about attributes beyond its interaction with $\otimes$-sequences. The same is true of missing zones. Having considered $\otimes$-sequences, shading, and missing zones, we are in a position to define the $\mathcal{I N}$-diagram that is a respectful translation of a $\mathcal{E} \mathcal{X}$-diagram.

Definition 12. Let $d_{\mathcal{E X}}=\left(E, Z_{E}, Z_{E}^{*}, S_{E}\right)$ and $d_{\mathcal{I N}}=\left(I, Z_{I}, Z_{I}^{*}, S_{I}\right)$ be Euler diagrams formed over $\mathcal{E} \mathcal{X}$ and $\mathcal{I} \mathcal{N}$ respectively. We say that $d_{\mathcal{I N}}$ is the respectful translation of $d_{\mathcal{E} \mathcal{X}}$ given the intensional label allocation function, Int, provided:

1. the intensional labels in $d_{\mathcal{I N}}$ arise from the extensional labels in $d_{\mathcal{E} \mathcal{X}}$ :

$$
I=\{\operatorname{Int}(p): p \in E\} .
$$

2. there are no missing zones in $d_{\mathcal{I N}}: \mathcal{M Z}\left(d_{\mathcal{I N}}\right)=\emptyset$.

3. there are no shaded zones in $d_{\mathcal{I N}}: Z_{I}^{*}=\emptyset$.

4. the $\otimes$-identifiers in $d_{\mathcal{I N}}$ arise from those in $d_{\mathcal{E X}}$ :

$$
S_{I}=\left\{r_{\mathcal{I N}} \subseteq Z_{I}: \exists r_{\mathcal{E X}} \in S_{E} r_{\mathcal{I N}} \in R_{\mathcal{I N}}\left(r_{\mathcal{E} \mathcal{X}} \backslash Z_{E}^{*}\right)\right\} .
$$

In figure $9, d_{\mathcal{I N}}$ is the respectful translation of $d_{\mathcal{E} \mathcal{X}}$. Likewise, $d_{\mathcal{I N}}^{\prime}$ is the respectful translation of $d_{\mathcal{E} \mathcal{X}}^{\prime}$ in figure 10 . Interestingly, $d_{\mathcal{I N}}$, figure 9 , is the respectful translation of $d_{\mathcal{E} \mathcal{X}}^{\prime \prime}$ in figure 11. This illustrates that the translation from extensional diagrams to intensional diagrams inherently loses information. Theorem 1, below, establishes that respectful translations ensure, given any model for $d_{\mathcal{E} \mathcal{X}}$ that any interpretation which is respectful of it is a model for $d_{\mathcal{I N}}$; the theorem is illustrated in figure $12^{3}$.

${ }^{3}$ Proofs of Theorem 1 and of Theorem 2 are omitted for reasons of space but can be found in an appendix on our website at http://readableproofs.org/looking-glass 


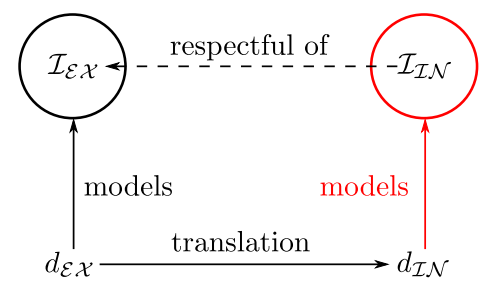

Fig. 12. The relationship between models.

Theorem 1. Let $d_{\mathcal{E X}}=\left(E, Z_{E}, Z_{E}^{*}, S_{E}\right)$ be an Euler diagram formed over $\mathcal{E} \mathcal{X}$. Let $d_{\mathcal{I N}}=\left(I, Z_{I}, Z_{I}^{*}, S_{I}\right)$ be a respectful translation of $d_{\mathcal{E X}}$. Let $\mathcal{I}_{\mathcal{E X}}=$ $\left(\mathcal{U}_{\mathcal{E X}}, \Psi_{\mathcal{E X}}\right)$ be a model for $d_{\mathcal{E X}}$. Let $\mathcal{I}_{\mathcal{I N}}=\left(\mathcal{U}_{\mathcal{I N}}, \Psi_{\mathcal{I N}}\right)$ be an interpretation over $\mathcal{I N}$ that is respectful of $\mathcal{I}_{\mathcal{E X}}$. Then $\mathcal{I}_{\mathcal{I N}}$ is a model for $d_{\mathcal{I N}}$.

\section{From Intensional to Extensional Diagrams}

Our task now is to consider what, if any, information we can derive about sets of individuals from information about attributes. We start by focusing on figure 13 . We see that there is an attribute in $A$ that is not in $B$. However, this does not imply that there are any individuals with that attribute: the presence of attributes tells us nothing about the presence of individuals. By contrast, the absence of attributes does provide information about the absence of individuals. In our example, the shading inside $B$ but outside $A$ intuitively tells us that the attributes in $A$ include all of those in $B$. Therefore, any individual with all of the attributes in $A$ also has all of the attributes in $B$, so any such individual must also be in the set $\operatorname{Ext}(B)$. This implies that $\operatorname{Ext}(A)$ is a subset of $\operatorname{Ext}(B)$, as indicated by the shading in $d_{\mathcal{E} \mathcal{X}}$.
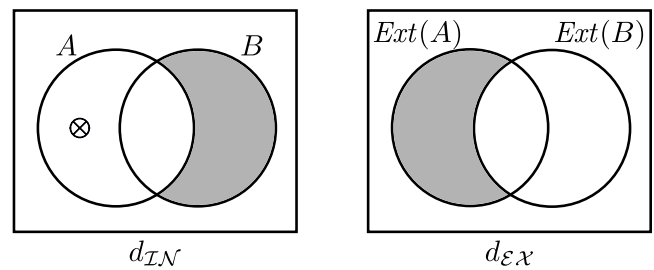

Fig. 13. A respectful translation from intensional to extensional diagrams.

Having established that $\otimes$-sequences in $\mathcal{I} \mathcal{N}$-diagrams provide no information about individuals in $\mathcal{E} \mathcal{X}$-diagrams, our focus is now exclusively on shading and missing zones. In figure $14, d_{\mathcal{I N}}^{\prime}$ contains three shaded zones. From the shading in $(\{A\},\{B, C\})$ we can see that all attributes in $A$ are all in $B$ or $C$. This implies that any individual in both $\operatorname{Ext}(B)$ and $\operatorname{Ext}(C)$ has all attributes in $A$. 

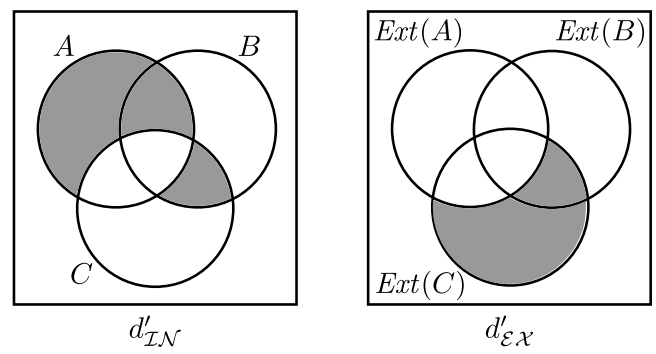

Fig. 14. A more complex shading case.

This insight allows us to shade the zone $(\{\operatorname{Ext}(B), \operatorname{Ext}(C)\},\{\operatorname{Ext}(A)\})$. Being in just one of $\operatorname{Ext}(B)$ and $\operatorname{Ext}(C)$ need not imply membership of $\operatorname{Ext}(A)$, however. Consider next the shaded zone $(\{A, B\},\{C\})$. Taking this shaded zone in isolation tells us that individuals with the common attributes of $A$ and $B$ possess all attributes in $C$. From this we cannot infer anything about the absence of individuals in the sets represented by zones of $d_{\mathcal{E} \mathcal{X}}^{\prime}$. A little more formally, from this shaded zone in $d_{\mathcal{I N}}^{\prime}$, any individual, $i$, where

$$
\operatorname{att}(i) \supseteq \Psi_{\mathcal{I N}}(A) \cap \Psi_{\mathcal{I N}}(B)
$$

ensures $\operatorname{att}(i) \supseteq \Psi_{\mathcal{I N}}(C)$. But there is no zone in $d_{\mathcal{E} \mathcal{X}}^{\prime}$ whose individuals are guaranteed to have all of the attributes common to both $A$ and $B$. This is because individuals in a zone, say $\left(L_{i}, L_{o}\right)$, in $d_{\mathcal{E} \mathcal{X}}^{\prime}$, have all of the attributes in

$$
\bigcup_{p \in L_{i}} \Psi(\operatorname{Int}(p))
$$

as opposed to an intersection of attribute sets. From this it follows that this shaded zone does not (in isolation) give rise to shading in $d_{\mathcal{E} \mathcal{X}}^{\prime}$.

However, if we consider this shaded zone together with the shaded zone $(\{A\},\{B, C\})$, we form a zonal region, namely $\langle\{\{A\},\{C\},\{A, B, C\}\rangle$. This shaded zonal region tells us that all individuals with attributes in $A$ have all attributes in $C$ : there cannot be $\operatorname{Ext}(C)$ individuals that are not $\operatorname{Ext}(A)$ individuals. This allows us to shade the zonal region

$$
\langle\{\operatorname{Ext}(C)\},\{\operatorname{Ext}(A)\},\{\operatorname{Ext}(A), \operatorname{Ext}(B), \operatorname{Ext}(C)\}\rangle
$$

(of course, some of this shading in $d_{\mathcal{E} \mathcal{X}}^{\prime}$ was already obtained from the shading in $(\{A\},\{B, C\}))$. Lastly, just as taking $(\{A, B\},\{C\})$ in isolation did not yield information in $d_{\mathcal{E} \mathcal{X}}$, the shading in $\left.(\{B, C\},\{A)\}\right)$ gives rise to no information about individuals. In summary, we gain information about the absence of individuals in $\mathcal{E} \mathcal{X}$-diagrams is when $\mathcal{I N}$-diagrams contain shading in zonal regions whose $I N$ set contains a single label.

For our final example in the build-up to defining a respectful translation from $\mathcal{I N}$-diagrams to $\mathcal{E} \mathcal{X}$-diagrams, we consider figure 15 . Here, there is one shaded 

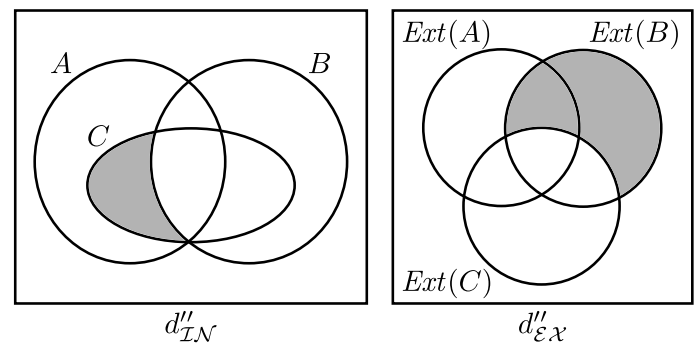

Fig. 15. The impact of missing zones.

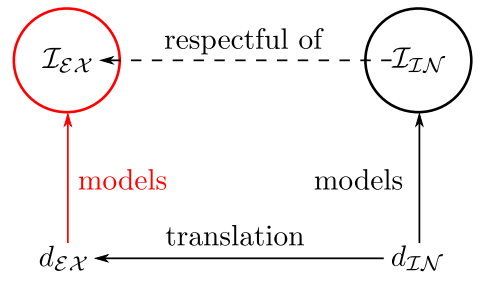

Fig. 16. The relationship between models.

zone in $d_{\mathcal{I} \mathcal{N}}^{\prime \prime}$ and also one missing zone. These two zones form a zonal region, namely $\langle\{C\},\{B\},\{A, B, C\}\rangle$. Consistent with our earlier examples, this gives rise to the shading in $d_{\mathcal{E X}}$. This leads to our next definition:

Definition 13. Let $\langle I N, O U T, I\rangle$ be a zonal region formed over $\mathcal{I N}$ such that $|I N|=1$. Let $\left\langle I N^{\prime}, O U T^{\prime}, E\right\rangle$ be a zonal region formed over $\mathcal{E} \mathcal{X}$. Then $\left\langle I N^{\prime}, O U T^{\prime}, I\right\rangle$ is a corresponding $\mathcal{E} \mathcal{X}$-region of $\langle I N, O U T, L\rangle$, denoted

$$
\langle I N, O U T, I\rangle \equiv_{c}\left\langle I N^{\prime}, O U T^{\prime}, E\right\rangle
$$

provided

1. IN ${ }^{\prime}$ contains a label arising from OUT: $I N^{\prime}=\{\operatorname{Ext}(a): a \in O U T\}$,

2. OUT $T^{\prime}$ contains the label arising from IN:OUT $T^{\prime}=\{\operatorname{Ext}(a): a \in I N\}$, and

3. E contains the intensional labels arising from $I: E=\{\operatorname{Ext}(a): a \in I\}$.

We are now in a position to define a respectful translation from $\mathcal{I N}$-diagrams to $\mathcal{E} \mathcal{X}$-diagrams:

Definition 14. Let $d_{\mathcal{I N}}=\left(I, Z_{I}, Z_{I}^{*}, S_{I}\right)$ and $d_{\mathcal{E X}}=\left(E, Z_{E}, Z_{E}^{*}, S_{E}\right)$ be Euler diagrams formed over $\mathcal{I N}$ and $\mathcal{E} \mathcal{X}$ respectively. We say that $d_{\mathcal{E} \mathcal{X}}$ is the respectful translation of $d_{\mathcal{I N}}$ given the intensional label allocation function Int provided:

1. the extensional labels in $d_{\mathcal{E X}}$ arise from the intensional labels in $d_{\mathcal{I N}}: E=$ $\{\operatorname{Ext}(a): a \in I\}$.

2. there are no missing zones in $d_{\mathcal{E} X}: \mathcal{M Z}\left(d_{\mathcal{E X}}\right)=\emptyset$.

3. the shaded zones $d_{\mathcal{E} \mathcal{X}}$ arise from some shaded and missing zones in $d_{\mathcal{I N}}$ :

$$
\begin{gathered}
Z_{E}^{*}=\left\{z_{E} \in\left\langle I N^{\prime}, O U T^{\prime}, E\right\rangle: \exists\langle I N, O U T, I\rangle \subseteq Z_{I}^{*} \cup \mathcal{M Z}\left(d_{I}\right)\right. \\
\left.|I N|=1 \wedge\langle I N, O U T, I\rangle \equiv_{c}\left\langle I N^{\prime}, O U T^{\prime}, E\right\rangle\right\},
\end{gathered}
$$

4. there are no $\otimes$-identifiers in $d_{\mathcal{E} \mathcal{X}}: S_{E}=\emptyset$.

In figure $13, d_{\mathcal{I N}}$ is the respectful translation of $d_{\mathcal{E} \mathcal{X}}$, which illustrates that the translation from $\mathcal{I N}$-diagrams to $\mathcal{E} \mathcal{X}$-diagrams inherently loses information 
provided by $\otimes$-sequences. We have a similar situation with shading, where $d_{\mathcal{I N}}^{\prime}$ is the respectful translation of $d_{\mathcal{E} \mathcal{X}}^{\prime}$ in figure 14; here the shading could only be partially translated. Lastly, $d_{\mathcal{I} \mathcal{N}}^{\prime \prime}$ in figure 15 highlights the role of missing zones when respectfully translating to $d_{\mathcal{E} \mathcal{X}}$. Theorem 2 , below, establishes that respectful translations ensure that models for $d_{\mathcal{I N}}$ respect only interpretations which are models for $d_{\mathcal{E} \mathcal{X}}$; the theorem is illustrated in figure 16 .

Theorem 2. Let $d_{\mathcal{I N}}=\left(I, Z_{I}, Z_{I}^{*}, S_{I}\right)$ be an Euler diagram formed over $\mathcal{I N}$. Let $d_{\mathcal{E X}}=\left(E, Z_{E}, Z_{E}^{*}, S_{E}\right)$ be a respectful translation of $d_{\mathcal{I N}} \cdot$ Let $\mathcal{I}_{\mathcal{I N}}=\left(\mathcal{U}_{\mathcal{I N}}, \Psi_{\mathcal{I N}}\right)$ be a model for $d_{\mathcal{I N}}$. Let $\mathcal{I}_{\mathcal{E} \mathcal{X}}=\left(\mathcal{U}_{\mathcal{E X}}, \Psi_{\mathcal{E} \mathcal{X}}\right)$ be an interpretation over $\mathcal{E} \mathcal{X}$ such that $\mathcal{I}_{\mathcal{I N}}$ is respectful of $\mathcal{I}_{\mathcal{E} \mathcal{X}}$. Then $\mathcal{I}_{\mathcal{E} \mathcal{X}}$ is a model for $d_{\mathcal{E} \mathcal{X}}$.

We now move on to summarise the results of the paper and look forward to possible directions in which it could be taken.

\section{Conclusion}

In this paper we have formalised the idea of extensional and intensional Euler diagrams, providing a systematic study of the extent and intent of a term. We established several basic results about the relationship between the two perspectives; theorems 1 and 2 demonstrate the symmetry of the respectfulness relation, used to give the definitions of the translations in both directions between extent and intent. In essence our translations maintain the (minimal) information which must be true in either perspective. These results show the inevitability of information loss when translating from one system to the other.

Concerning information loss, it will be interesting to precisely characterise its nature in future work. In this context, we envisage defining an equivalence relation that syntactically characterises when two extensional diagrams (resp. intensional diagrams) give rise the same intensional diagram. Clearly, two extensional diagrams which differ only in their missing zones and shading give rise to the same intensional diagram (resp. extensional diagram). In figure $17, d_{\mathcal{E} \mathcal{X} 1}$ and $d_{\mathcal{E} \mathcal{X}_{2}}$ differ in this way and both translate to $d_{\mathcal{I N}}$. In addition, $d_{\mathcal{E} \mathcal{X} 3}$, which contains an additional $\otimes$ in shaded zone, also gives rise to $d_{\mathcal{I N}}$. This is because shaded zones always represent the empty set, even if they contain an $\otimes$ symbol.

The inspiration for exploring the relationship between extensional and intensional Euler diagrams came from a thought experiment in Moktefi's 2015 paper [7]. The subject of that article is the "iconicity" of using circles to represent extent, a topic beyond scope for this discussion. However, iconicity depends on resemblances between notation and meaning; the kind of resemblance involved in this case is said to be between spatial relations of circles (e.g. one within another, two placed apart) and relations of sets (e.g. subsumption, disjointness). That is, the article ([7]) argues that circles are iconic to extents of terms because circles have the relations that sets do and that we want to depict. The thought experiment involves a hypothetical intensional Euler diagram notation without defining it, and illustrates that Euler diagrams are less iconic (i.e. do not possess the relevant relations) when used to depict the intent of terms. 


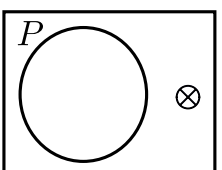

$d_{\mathcal{E} \mathcal{X} 1}$

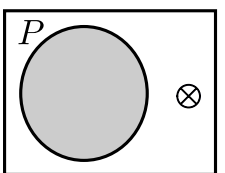

$d_{\mathcal{E} \mathcal{X} 2}$

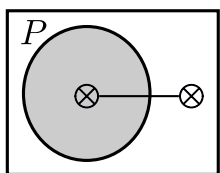

$d_{\mathcal{E} \mathcal{X} 3}$

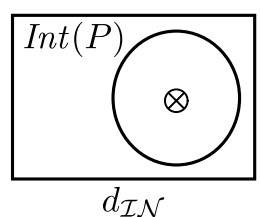

Fig. 17. Extensional diagrams translating to the same intensional diagram.

Although this paper does not do so, the purpose of creating this formalism is to carry on the work of the thought experiment: if we accept that a notation based on circles (or, generally, closed curves) arranged in space is an effective language for reasoning about extent, what happens to this effectiveness when we use a similar notation to reason about intent? What, if anything, does the transition from extensional to intensional spatial diagrams tell us about the "effectiveness" (whether explained as iconicity, well-matchedness or using other terminology) of using space to depict extent? What form would a notation take which "has" the salient relations of intension? Would translating from extensional Euler diagrams to such an intensional notation involve the necessary loss of information? These open questions can be considered from numerous points of view, and the work we have presented is the first step towards a formal logical perspective.

\section{References}

1. Bassler, O.B.: Leibniz on intension, extension, and the representation of syllogistic inference. Synthese 2(116), 117-139 (1998)

2. Couturat, L.: La Logique de Leibniz. Félix Alcan (1901)

3. Dipert, R.R.: Individuals and extensional logic in schroder's 'vorlesungen uber die algebra der logik'. Modern Logic 2-3(1), 140-159 (1991)

4. Fitting, M.: Intensional logic. https://plato.stanford.edu/archives/sum2015/ entries/logic-intensional/ (2015), accessed December 2017

5. Hurley, P.J.: A Concise Introduction to Logic, 12th ed. Stamford (2015)

6. Lewis, C.I.: A Survey of Symbolic Logic. University of California Press (1918)

7. Moktefi, A.: Is Euler's circle a symbol or an icon? Sign Systems Studies 43(4), $597+($ Dec 2015)

8. Moktefi, A., Pietarinen, A.V.: On the Diagrammatic Representation of Existential Statements with Venn Diagrams. Journal of Logic, Language and Information 24(4), 361-374 (2015)

9. Shearman, A.T.: The Development of Symbolic Logic. Williams and Norgate (1906)

10. Shin, S.J.: The Logical Status of Diagrams. Cambridge University Press (1994)

11. Stapleton, G.: Delivering the potential of diagrammatic logics. In: International Workshop on Diagrams, Logic and Cognition. CEUR, vol. 1132, pp. 1-8 (2013), available at http://ceur-ws.org/Vol-1132/paper1.pdf

12. Venn, J.: On the diagrammatic and mechanical representation of propositions and reasonings. Phil.Mag (1880)

13. Venn, J.: Symbolic Logic. Macmillan (1894) 